\title{
Biofilm: Contributing Factor to Drug Resistance in Staphylococci in Blood Stream Infections
}

\author{
Aritlce by Sandeep Thirunavukkarasu, Rathish K C, Saroj Golia \\ M S Ramaiah Medical college \\ Email:-dr.sandeept@yahoo.co.in
}

\begin{abstract}
Staphylococci represent the most commonly encountered blood culture isolates from ICU patients. Slime production is considered to be a significant virulence factor for some strains of Staphylococci. The importance of the role played by slime is further increased by its frequent association to reduced antibiotic susceptibility. This study was done to find out relationship of Bio-Film formation on the susceptibility pattern of the isolates. 27.6\% of the isolates produced biofilm as detected by Congo Red Agar method; commonest being Staphylococcus epidermidis (53.9\%). The percentage of resistance in the group which produces biofilm is always greater than the group which does not produce biofilm. Odds ratio for overall drug resistance was 2.10 (with 95\% confidence interval as [1.6 - 2.6]) and with a p-value of 0.006.
\end{abstract}

Keywords: Biofilm, Staphylococcus, Blood stream infections

\section{Introduction}

Management of infections in ICU is one of the most challenging tasks for an intensivist in any hospital across the world; Blood stream infections (BSI) being the most common. It assumes the highest priority in terms of management due to high rates of patient morbidity and mortality; global rates of upto 40\% [1] have been estimated. Staphylococcus aureus, Escherichia coli, Coagulase-negative Staphylococcus (CoNS), Klebsiella pnemoniae \& Enterococci [2] are the 5 commonly detected bacteria in blood of septicemic patients. Staphylococcus aureus bacteremia is a serious infection associated with high morbidity and mortality because of its ability to cause metastatic lesions and persistent bacteremia. The risk factors for persistence include endovascular sources, cardiovascular prosthesis, metastatic infections, Vancomycin treatment, diabetes and biofilm production. Few organisms like CoNS are characterized by an ability to colonize the surfaces of biomaterials by adhering in biofilmstructured communities of cells encased in a self-produced polymeric matrix, an amorphous slimy material that is loosely bound to Staphylococcal cells. Slime is believed to make the micro-organisms more resistant to administered antibiotics and to host-defense mechanisms. Production of slime is characteristic of many strains of $S$. epidermis and $S$. aureus.

It is well known that slime production plays an important role in the pathogenesis of infections caused by different micro organisms especially Staphylococci. But very few studies have been conducted on isolates from human samples to find out the association between drug resistance and biofilm production. This study was conducted to estimate the percentage of Staphylococci producing biofilm and its association with drug resistance. 
South American Journal of Medicine

Special Edition 2016

\section{Methods and materials}

It was a prospective study conducted in a 800 bed tertiary care centre with 30 bed ICU. The target population was patients admitted to ICU with a suspicion of Staphylococcal bacteremia. The clinical diagnosis of bacteremia was based on criteria of Bates \& Herwaldt [3]. Two blood cultures were collected from each patient. After taking informed consent, $5 \mathrm{ml}$ of venous blood sample was collected under aseptic precautions and inoculated in automated blood culture bottles containing Trypticase soy broth with antibiotic adsorbing beads. Gram stain and subculture were done on the bottles which were flagged positive by the instrument. Staphylococci were identified using standard biochemical tests.

Biofilm production was detected by growing the isolates on $0.8 \%$ Congo red agar. A positive result was indicated by black colonies on the surface \& Non-slime producing strains developed red colonies after incubation $37^{\circ} \mathrm{C}$ for $24 \mathrm{~h}$. Susceptibility to antibiotics was determined by the Kirby Bauer disk diffusion method on Mueller-Hinton agar plates. Twelve antibiotics were chosen for the study supplied by Hi Media Laboratories, Mumbai, according to CLSI 2015 guidelines. The antibiotics used were Penicillin (10U), Amoxicillin-Clavulanic acid $(20 / 10 \mu \mathrm{g})$, Gentamicin $(10 \mu \mathrm{g})$, Cefotaxime $(30 \mu \mathrm{g})$, Cefoxitin $(30 \mu \mathrm{g})$, Linezolid $(30 \mu \mathrm{g})$, Ciprofloxacin $(5 \mu \mathrm{g})$, Tetracycline $(30 \mu \mathrm{g})$, Co-trimoxazole $(25 \mu \mathrm{g})$, Clindamycin $(2 \mu \mathrm{g})$, Erythromycin $(15 \mu \mathrm{g})$, Teicoplanin $(30 \mu \mathrm{g})$.

To check the association between biofilm production and antibiotic resistance, odds ratio was used. The odds ratio is a measure of effect size, describing the strength of association or non-independence between two binary data values.

\section{Results}

460 patients satisfied the inclusion criteria from whom 2 to $5 \mathrm{ml}$ of venous blood sample was collected and subjected to further tests. 94 of them yield growth of Gram Positive Cocci in clusters (suggesting Staphylococci) and were considered for further analysis. The following figure (Figure 1) shows the different species isolated

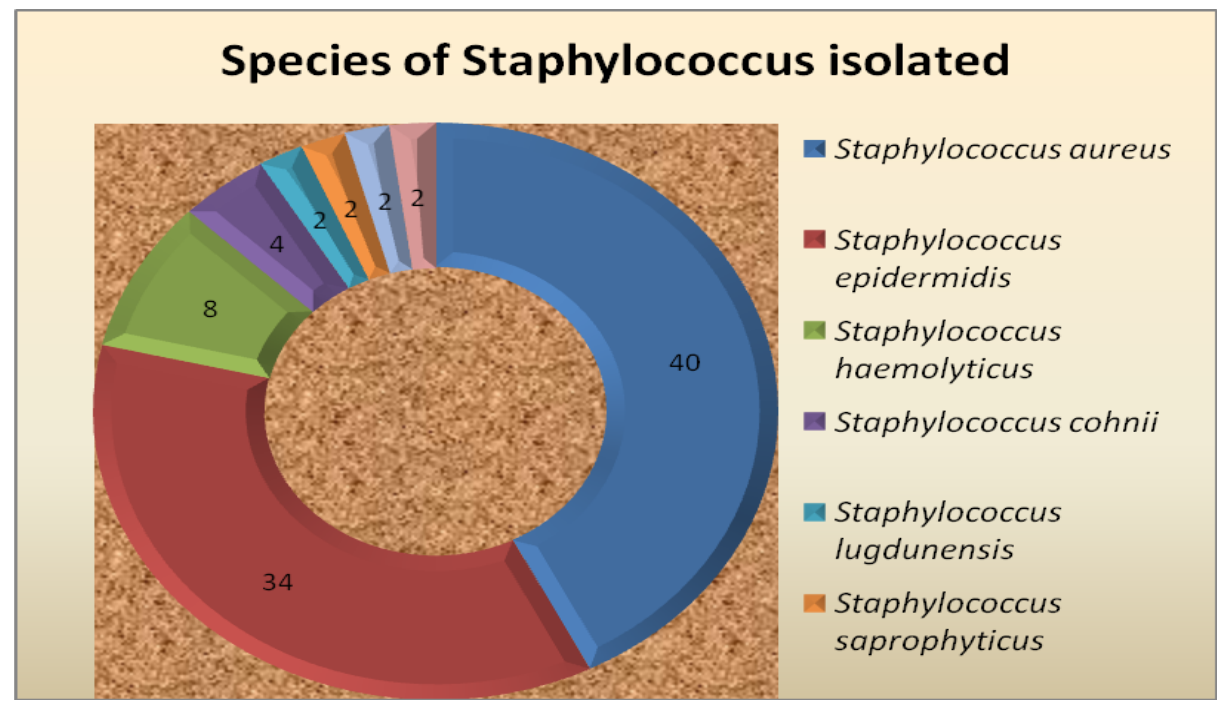

Figure 1 - Species of staphylococci isolated

Bio-film formation was detected using Congo Red Agar. There were 26 (27.6\%) isolates which produced bio-film. The following table (Table 1) gives details on different species of Staphylococci producing biofilms. 
Table 1 - Species of staphylococci producing bio-film

\begin{tabular}{|l|c|c|}
\hline Species & Number & Percentage \\
\hline Staphylococcus aureus & 8 & 30.7 \\
\hline Staphylococcus epidermidis & 14 & 53.9 \\
\hline Staphylococcus haemolyticus & 2 & 7.7 \\
\hline Staphylococcus cohnii & 2 & 7.7 \\
\hline
\end{tabular}

The following graph (Figure 2) demonstrates the drug resistance with respect to biofilm production. The percentage of resistance in the group which produces bio-film is greater than the group which does not produce bio-film.

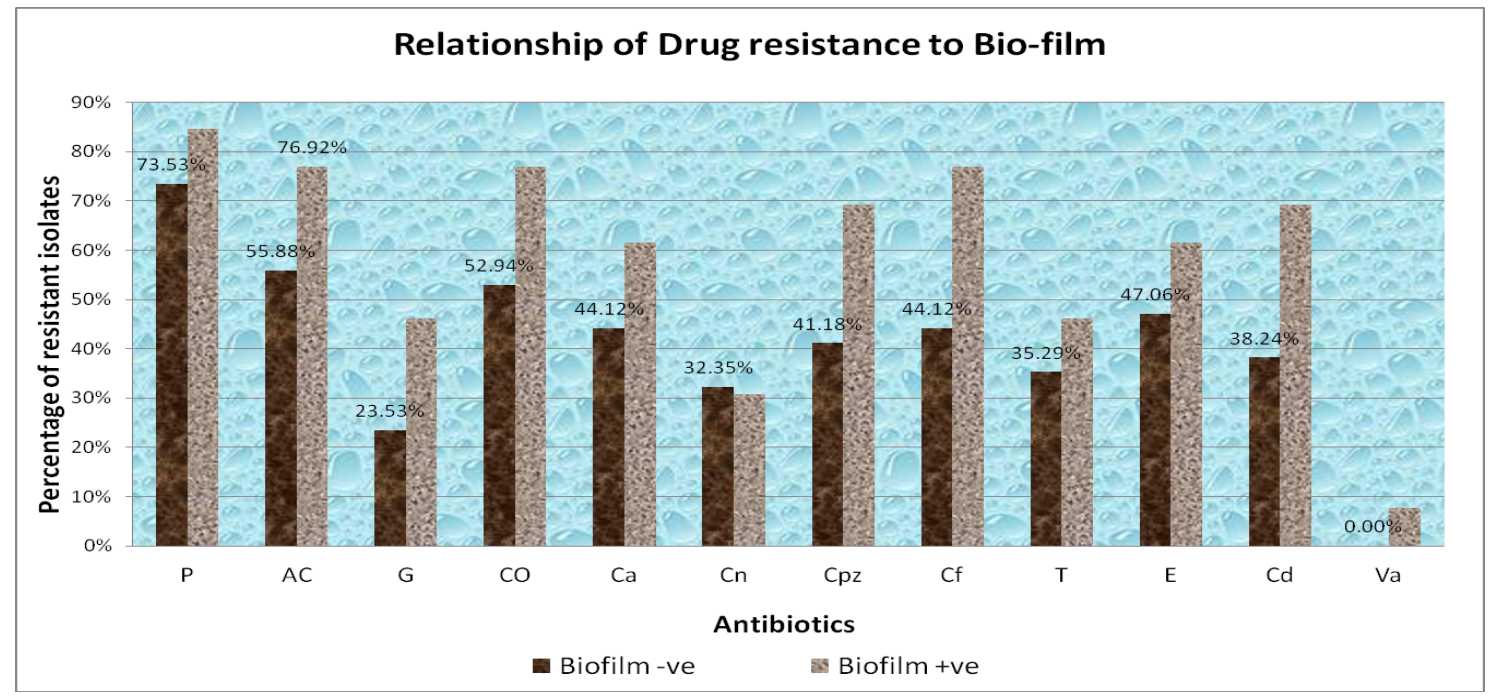

Figure 2 - Relationship of drug resistance to bio-film

However, significant increase in drug resistance is seen only in Co-trimoxazole, Cefoperazone, Ciprofloxacin and Clindamycin. $(\mathrm{p}<0.005)$ Odds ratio for overall drug resistance was 2.10 (with $95 \%$ confidence interval as $[1.6-2.6]$ ) and with a p-value of 0.006 . This implies that the organism is 2.1 times more resistant to antibiotics if it produces bio-film. Odds ratio was calculated for individual drugs and the results are as follows (Table 3)

Table 3 - Odds ratio for different antibiotics

\begin{tabular}{|l|c|}
\hline Drug & Odds ratio \\
\hline Penicillin & 1.98 \\
\hline Amoxy Clav & 2.63 \\
\hline Gentamicin & 2.79 \\
\hline Co-trimoxazole & 2.76 \\
\hline Cefoxitin & 0.93 \\
\hline Cefoperazone & 3.21 \\
\hline Ciprofloxacin & 4.22 \\
\hline
\end{tabular}


South American Journal of Medicine

Special Edition

\begin{tabular}{|l|l|}
\hline Tetracycline & 1.57 \\
\hline Erythromycin & 1.80 \\
\hline Clindamycin & 3.63 \\
\hline
\end{tabular}

The odds ratio of most of the antibiotics is much higher than 1, indicating that drug resistance is more likely to occur in the bio-film producing group. The exception is Cefoxitin.

\section{Discussion}

Blood stream infections (BSI) are one of the most common nosocomial infections encountered in hospital especially in ICU patients, less so in ward patients. In the current study, a total of 460 patients were screened, out of which, 94 (20\%) positive blood cultures were identified as Staphylococcal isolates by using the standard cultural and identification methods. This is in alignment with the prevalence rate as reported by Aygen and associates [4] and Mathur and colleagues [5]

Slime production plays an important role in the pathogenesis of infections caused by different microorganisms, especially staphylococci. In this study all the species of staphylococci produced bio-film except for S. lugdunensis, S. capitis and S. warneri. The data reported here indicate an important role of slime production as a virulence marker for clinically significant $S$. epidermidis isolates. The results are similar to those reported by other authors [6], who found that $S$. epidermidis frequently causes nosocomial septicemia, and infects indwelling medical devices like intravascular catheters and prosthetic valves. However, the findings of the present study showed that slime formation was not more prominent in $S$. aureus strains than in CNS strains isolated.

The odds ratio is the ratio of the odds of an event occurring in one group to the odds of it occurring in another group. The measure describes the strength of association or non-independence between two binary data values. In this case the groups are defined by the production and non-production of bio-film. An odds ratio of 1 indicates that the resistance to drug under study is equally likely to occur in the groups, i.e, bio-film producing and not producing. An odds ratio greater than 1 indicates that the resistance to drug more likely to occur in the bio-film producing group. And an odds ratio less than 1 indicates that the resistance to drug is less likely to occur in the bio-film producing group.

In this study it was found that the organism is 2.1 times more resistant to antibiotics if it produces bio-film. The odds ratio ranges from 1.57 (Tetracycline) to a high value of 4.22 (Ciprofloxacin). This indicates that existing drugs may be ineffective in treating isolates producing bio-films involved in bacteremia. This has been found by other studies also which involved prosthetic device related infections[7].

Unlike all other antibiotics, Cefoxitin gives an odds ratio of 0.93 which is less than 1. This indicates that susceptibility to this antibiotic is not affected by production of biofilm. In 2009, CLSI guidelines changed the method of choice to detect MRSA. They recommended Cefoxitin disc diffusion technique. Susceptibility to Cefoxitin not being affected by biofilm may be considered as an advantage to continue the use of this drug to detect MRSA. Kotulova [8] in his paper has discussed that organism producing biofilm is highly resistant to Gentamicin. This study also confirms this finding with an odds ratio of 2.79 . 


\section{Conclusion}

$27.6 \%$ of the Staphylococcal isolates produced bio-film as detected by Congo Red Agar method. Bio-film production was seen maximum in S. epidermidis $(53.9 \%)$ followed by $S$. aureus (30.7\%). The percentage of resistance is always greater in the isolate which produces bio-film than isolate which does not produce bio-film with an odds ratio of 2.10. Significant increase in resistance is seen only to Cotrimoxazole, Cefoperazone, Ciprofloxacin and Clindamycin.

\section{References}

[1]. Morales Serrano T, Ramos S, Lara Gonzalez Y, Torres Colberg H, Vera Quiñones A, Miranda Santiago R, Amill S, Otero M, Cintron V, Villarreal Morales ML, ' Manatí Medical Center Sepsis Management Epidemiological Study', Bol Asoc Med P R (2015), Vol.107 No.2 pp.34-39.

[2]. Weinstein M P, Towns M L, Quartey S M, Mirrett S, Reimer L G, Parmigiani G, 'The clinical significance of positive blood cultures in the 1990s: a prospective comprehensive evaluation of the microbiology, epidemiology, and outcome of bacteremia and fungemia in adults', Clin Infect Dis. (1997), Vol.24 No.4 pp.584-602.

[3]. Bates D W, Sands K, Miller E, 'Predicting bacteremia in patients with sepsis syndrome. Academic Medical Center Consortium Sepsis Project Working Group', J Infect Dis (1997), Vol.176 pp.1538-1551.

[4]. Aygen B, Yoruk A, Yyldyz O, 'Bloodstream infections caused by Staphylococcus aureus in university hospital in Turkey: clinical and molecular epidemiology of methicillin-resistant Staphylococcus aureus', Clin Microbiol. Infect. (2004), Vol.10 pp.309-314.

[5]. Mathur P, Kapil A, Dan B, 'Nosocomial bacteremia in intensive care unit patients of a tertiary care center', Indian J Med Res. (2005), Vol.122 pp.305-308.

[6]. Ammendolia MG, Di Rosa R, Montanaro R, Arciola CR, Baldassarri L, 'Slime production and expression of the slime-associated antigen by Staphylococal clinical isolates', J Clin Microbiol (1999) Vol.37 pp.3235-3238.

[7]. Amorena B, Garcia E, Monzon M, Leiva J, Oteiza C, Perez M, 'Antibiotic susceptibility assay for Staphylococcus aureus in biofilms developed in-vitro', J. Antimicrob. Chemother. (1999), Vol.44 pp.43 - 55.

[8]. Kotulová D, Slobodníková L, 'Susceptibility of Staphylococcus aureus biofilms to Vancomycin, Gentamicin and Rifampin', Epidemiol Mikrobiol Imunol. (2010), Vol.59 No.2 pp.80-87. 